\title{
Neutralizing antibodies against the SARS-CoV-2 Delta and Omicron variants following heterologous CoronaVac plus BNT162b2 booster vaccination
}

\author{
Eddy Pérez-Then ${ }^{1,15}$, Carolina Lucas ${ }^{2,15} \bowtie$, Valter Silva Monteiro ${ }^{2,15}$, Marija Miric ${ }^{3,15}$, \\ Vivian Brache ${ }^{4}$, Leila Cochon ${ }^{4}$, Chantal B. F. Vogels ${ }^{5}{ }^{5}$, Amyn A. Malik ${ }^{6,7}$, Elena De la Cruz ${ }^{4}$, \\ Aidelis Jorge ${ }^{4}$, Margarita De los Santos ${ }^{4}$, Patricia Leon ${ }^{8}$, Mallery I. Breban ${ }^{5}{ }^{5}$, Kendall Billig ${ }^{5}$, \\ Inci Yildirim ${ }^{5,6,9}$, Claire Pearson ${ }^{10}$, Randy Downing ${ }^{10}$, Emily Gagnon ${ }^{10}$, Anthony Muyombwe ${ }^{10}$, \\ Jafar Razeq ${ }^{10}$, Melissa Campbell ${ }^{9}$, Albert I. Ko ${ }^{5,7,11}$, Saad B. Omer ${ }^{5,6,7}$, Nathan D. Grubaugh ${ }^{5,12}$, \\ Sten H. Vermund ${ }^{13}{ }^{13}$ and Akiko Iwasaki $\mathbb{B}^{1,14} \bowtie$
}

\begin{abstract}
The recent emergence of the SARS-CoV-2 Omicron variant is raising concerns because of its increased transmissibility and its numerous spike mutations, which have the potential to evade neutralizing antibodies elicited by COVID-19 vaccines. Here we evaluated the effects of a heterologous BNT162 b2 mRNA vaccine booster on the humoral immunity of participants who had received a two-dose regimen of CoronaVac, an inactivated vaccine used globally. We found that a heterologous CoronaVac prime vaccination of two doses followed by a BNT162b2 booster induces elevated virus-specific antibody levels and potent neutralization activity against the ancestral virus and the Delta variant, resembling the titers obtained after two doses of mRNA vaccines. Although neutralization of Omicron was undetectable in participants who had received a two-dose regimen of CoronaVac, the BNT162b2 booster resulted in a 1.4-fold increase in neutralization activity against Omicron compared with the two-dose mRNA vaccine. Despite this increase, neutralizing antibody titers were reduced by 7.1-fold and 3.6-fold for Omicron compared with the ancestral strain and the Delta variant, respectively. These findings have immediate implications for multiple countries that previously used a CoronaVac regimen and reinforce the idea that the Omicron variant is associated with immune escape from vaccines or infection-induced immunity, highlighting the global need for vaccine boosters to combat the impact of emerging variants.
\end{abstract}

The ongoing evolution of severe acute respiratory syndrome coronavirus 2 (SARS-CoV-2) and the recent emergence of the
Omicron variant raise concerns about its increased transmissibility and vaccine effectiveness. CoronaVac is a two-dose $\beta$-propiolactoneinactivated, aluminum hydroxide-adjuvanted coronavirus disease 2019 (COVID-19) vaccine. CoronaVac is widely used globally and has been authorized in 48 countries, with $85 \%$ and $80 \%$ effectiveness against hospital admission and death, respectively ${ }^{1}$. However, with the emergence of new SARS-CoV-2 variants and the waning immunity of vaccines over time, multiple countries have initiated the use of booster doses ${ }^{2-4}$. The Dominican Republic was among the first countries to recommend a third booster dose to address potential waning immunity and reduced effectiveness against variants.

The Omicron variant contains up to 36 mutations in the spike protein $^{5}$, rendering many vaccines less effective ${ }^{6,7}$. The Omicron variant is also highly transmissible, overtaking Delta as the dominant variant in many countries. To assess the potential risk of vaccine immune evasion we assembled a cohort of CoronaVac-vaccinated individuals who had received a heterologous BNT162b2 mRNA vaccine boost. We investigated vaccine-induced neutralizing antibody titers against the Delta (B.1.617.2) and the Omicron (BA.1 sublineage of B.1.1.529) variants and compared them with the neutralization titers produced against the ancestral A lineage, using authentic SARS-CoV-2 isolates.

To characterize SARS-CoV-2-specific adaptive immune responses we analyzed plasma samples from 101 non-hospitalized adult participants who received the BNT162b2 booster dose between 30 July and 27 August 2021, at least 4 weeks after the second dose of CoronaVac. Plasma samples were collected longitudinally at Departamento de Investigaciones Biomedicas, Clinica

\footnotetext{
'Ministry of Health, Santo Domingo, Dominican Republic. ²Department of Immunobiology, Yale University School of Medicine, New Haven, CT, USA. ${ }^{3}$ Two Oceans in Health, Santo Domingo, Dominican Republic. ${ }^{4}$ Biomedical Research Department, Profamilia, Santo Domingo, Dominican Republic. ${ }^{5}$ Department of Epidemiology of Microbial Diseases, Yale School of Public Health, New Haven, CT, USA. ${ }^{6}$ Yale Institute for Global Health, Yale University, New Haven, CT, USA. ${ }^{7}$ Section of Infectious Diseases, Department of Medicine, Yale University School of Medicine, New Haven, CT, USA. ${ }^{8}$ Laboratorio de Referencia, Santo Domingo, Dominican Republic. ${ }^{9}$ Section of Infectious Diseases and Global Health, Department of Pediatrics, Yale University School of Medicine, New Haven, CT, USA. ${ }^{10}$ Connecticut State Department of Public Health, Rocky Hill, CT, USA. "1Instituto Gonçalo Moniz, Fundação Oswaldo Cruz, Salvador, Brazil. ${ }^{12}$ Department of Ecology and Evolutionary Biology, Yale University, New Haven, CT, USA. ${ }^{13}$ Yale School of Public Health, New Haven, CT, USA. ${ }^{14}$ Howard Hughes Medical Institute, Chevy Chase, MD, USA. ${ }^{15}$ These authors contributed equally: Eddy Pérez-Then, Carolina Lucas, Valter Silva Monteiro, Marija Miric.凶e-mail: carolina.lucas@yale.edu; akiko.iwasaki@yale.edu
} 

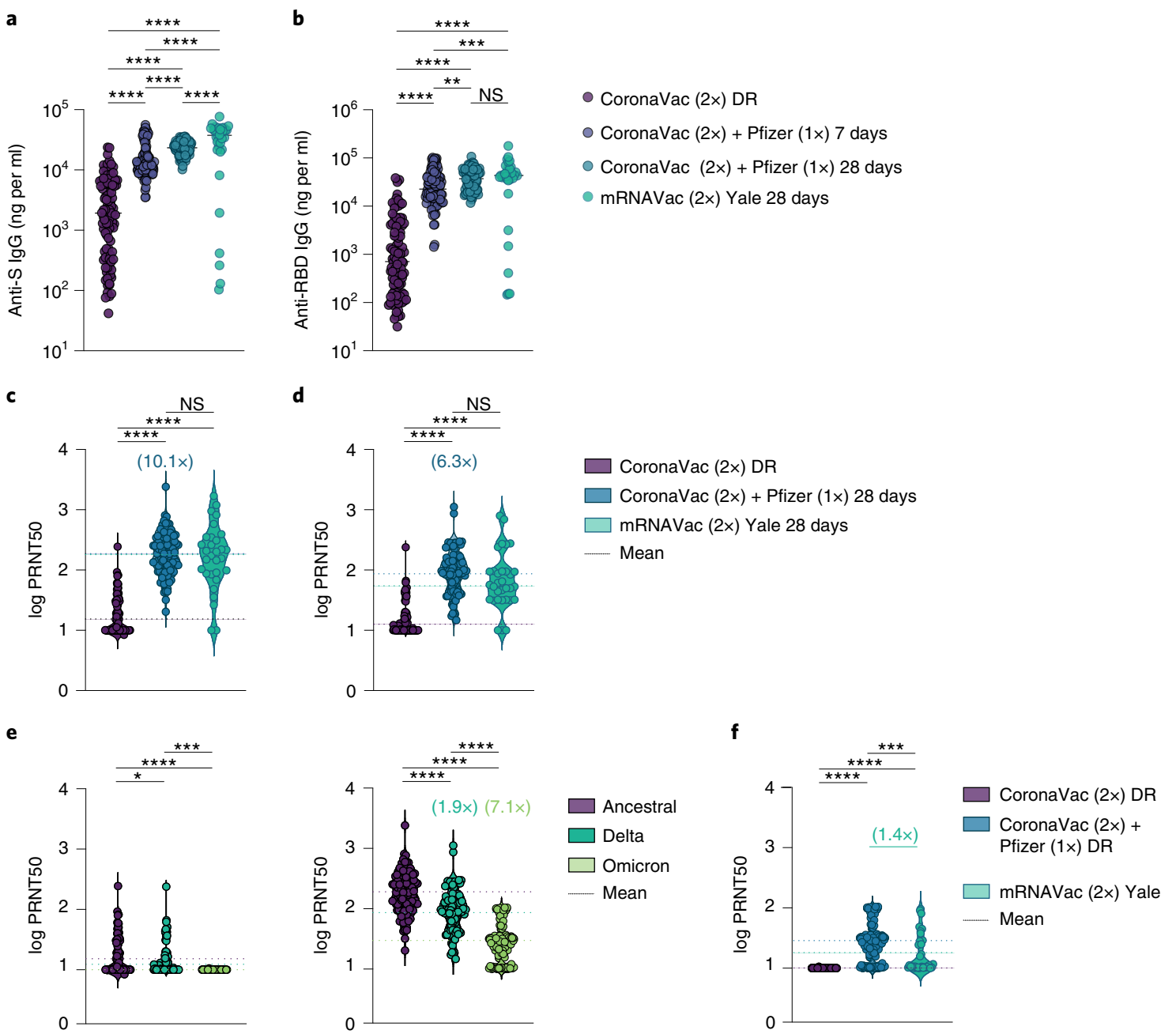

Fig. 1 | Characterization of vaccine-induced immunity after heterologous CoronaVac-BNT162b2 vaccination. Dominican Republic (DR) participants received two doses of CoronaVac followed by a heterologous booster with BNT162b2 mRNA vaccine. Plasma samples were collected at baseline, before the booster (CoronaVac (2x) DR), and at 7 and 28 days after the booster (CoronaVac (2x)+Pfizer (1x)). The HCW participants from Yale-New Haven Hospital received two doses of the mRNA vaccine and the plasma samples were used for comparison (mRNAVac ( $2 x$ ) Yale). a,b, Plasma reactivity to the spike (S) protein (a) and RBD (b) measured with ELISA (DR $n=101$ and Yale $n=32$ at each respective time point). Horizontal lines indicate the mean values. $\mathbf{c}-\mathbf{f}$, Neutralization assay using wild-type SARS-CoV-2. c,d, Plasma neutralization capacity against the ancestral strain (WA1, USA) (c) and the Delta variant (d) by time ( $D R, n=101$ in each group; Yale, $n=32$ ). The numbers in parentheses indicate the median fold change in neutralization resistance for the indicated variants compared with the ancestral strain. e, Plasma neutralization capacity against the ancestral strain and the Delta and Omicron variants at baseline (left) and at 28 days after the booster (right) ( $n=64$ for baseline Omicron variant, $n=101$ in other group). f, Plasma neutralization capacity against Omicron by time. CoronaVac (2x) DR, $n=64$; CoronaVac (2x)+Pfizer (1x) DR, $n=101$; mRNAVac (2x) Yale, $n=32$. Significance was assessed with one-way ANOVA corrected for multiple comparisons using Tukey's method. Violin plots represent the mean \pm s.d. Dotted lines indicate the mean and are colored accordingly. Each circle represents a single individual. ${ }^{\star} P<0.05$; ${ }^{\star \star} P<0.01$; ${ }^{\star \star \star} P<0.001$; ${ }^{\star \star \star \star} P<0.0001$; NS, not significant.

Evangelina Rodriguez, Profamilia, Santo Domingo, Dominican Republic, at baseline (prior to the booster), 7 and 28 days after the booster (third dose), and were analyzed with ELISA and neutralization assays using authentic virus. Data from a previous cohort, composed of healthcare workers (HCWs) from the Yale-New Haven Hospital who received two doses of an mRNA COVID-19 vaccine (mRNA-1273, Moderna or BNT162b2, Pfizer-BioNTech) were used as a reference ${ }^{8}$. The mean age of the 101 participants from the Dominican Republic (the majority of whom (70\%) were female) was 40.4 (s.d. 13.4) years and the body mass index was 27.6 (s.d. $5.5) \mathrm{kg} \mathrm{m}^{-2}$. Cohort demographics, vaccination and infection status are summarized in Extended Data Tables 1 and 2.

Plasma antibody reactivity to the spike protein and receptor binding domain (RBD) of SARS-CoV-2 were measured at baseline and at 7 and 28 days after the BNT162b2 booster.
Virus-specific immunoglobulin G (IgG) titers increased at 7 days above baseline, and were further elevated on day 28 after the booster shot (Fig. 1a,b). Individuals who received the CoronaVac prime vaccination of two doses followed by the BNT162b2 booster developed high anti-RBD IgG titers that reached equivalent levels to those of the HCWs who received two doses of mRNA vaccines.

We then measured the ability of serum samples to neutralize SARS-CoV-2, lineage A (ancestral strain, USA-WA1/2020) and lineage B.1.617.2 (Delta variant). Individuals who were fully vaccinated with CoronaVac and who had received the BNT162b2 booster had a 10.1- and 6.3-fold increase in neutralization activity against the ancestral strain and the Delta variant, respectively, 28 days after the booster shot. No statistically significant differences were observed in neutralization titers between individuals who 

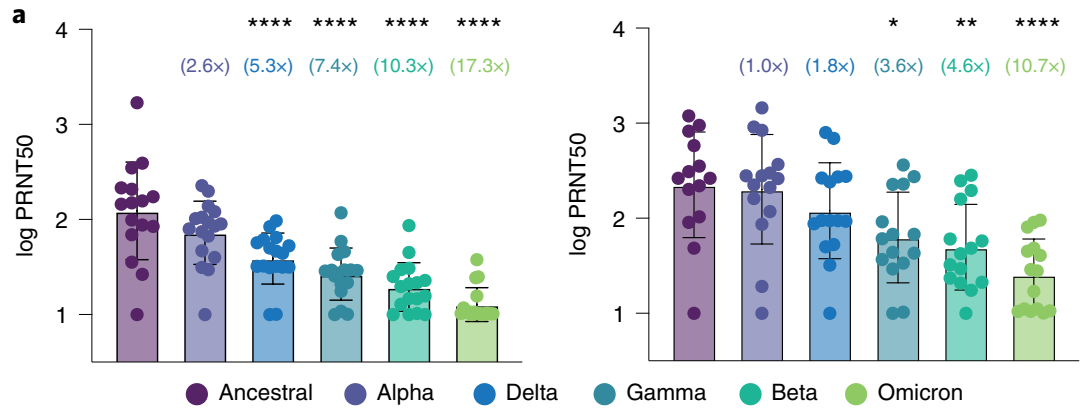

C

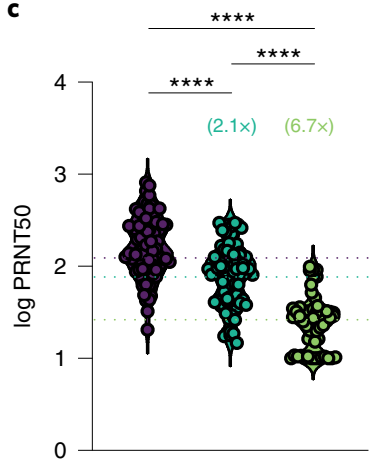

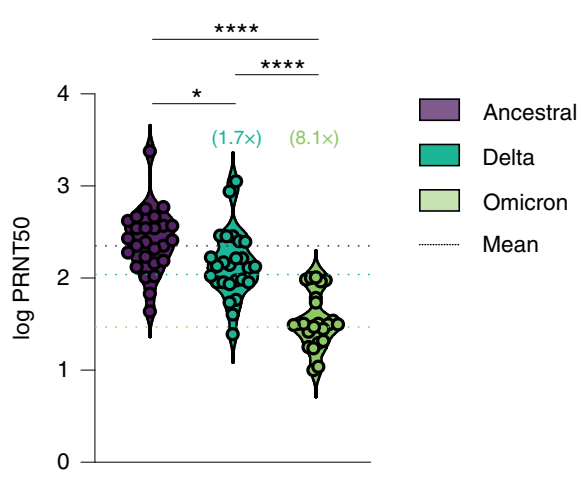

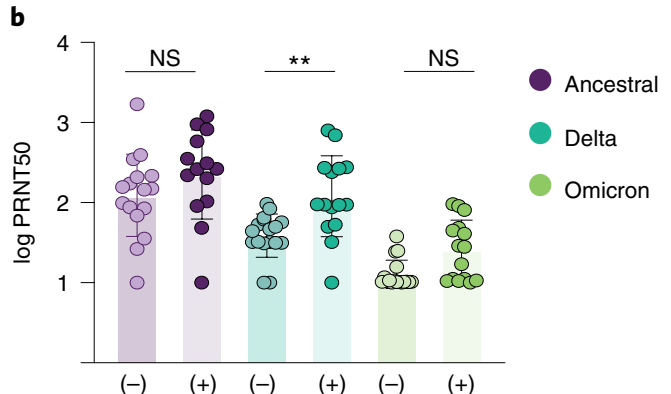

$(-) \quad(+) \quad(-) \quad(+) \quad(-) \quad(+)$

(-) Non-previously infected

(+) Previously infected

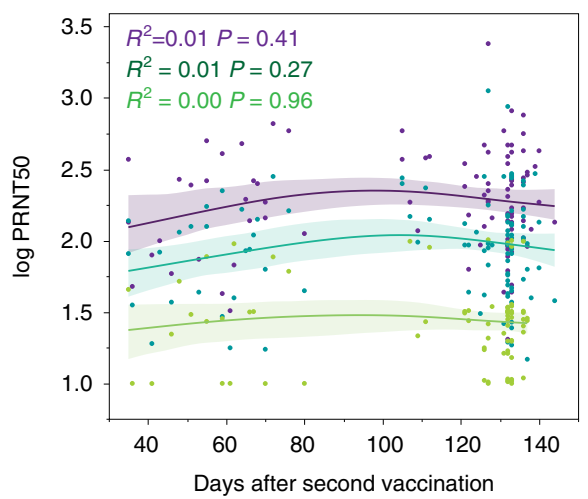

f

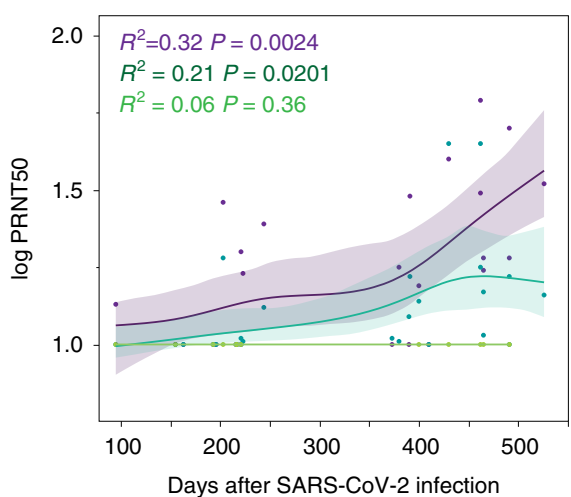

g

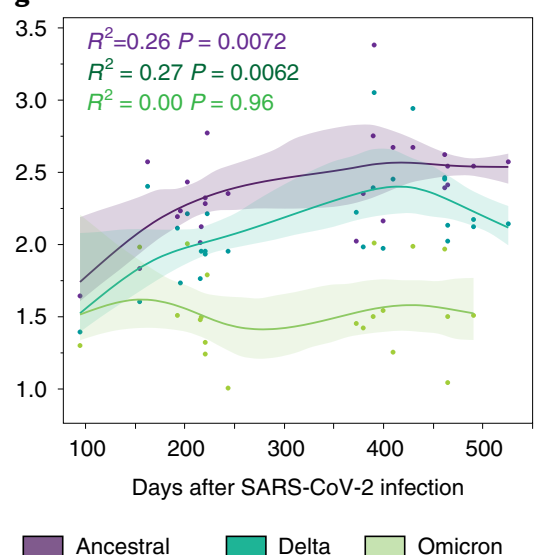

Fig. 2 | Comparison of neutralizing activity in CoronaVac-BNT162b2-vaccinated participants by SARS-CoV-2 infection status. a,b, Plasma neutralization titers against ancestral lineage A virus and variants of concern in HCW participants from Yale-New Haven Hospital who received two doses of the mRNA vaccine. a, Plasma neutralization titers measured at 28 days after the second dose in non-previously infected (left) and previously infected participants (right). Significance was measured using one-way ANOVA corrected using Dunnett's test. Boxes represent the mean \pm s.d. The numbers in parentheses indicate the median fold change in neutralization resistance for the indicated variants compared with the ancestral strain. $\mathbf{b}$, Neutralization titers from the participants in a by SARS-CoV-2 infection status. Significance was measured using one-way ANOVA corrected using Tukey's test. (-) Vaccinateduninfected, $n=16 ;(+)$ vaccinated-previously infected, $n=14$. $\mathbf{c}-\mathbf{g}$, Plasma neutralization titers from Dominican Republic participants who received two doses of CoronaVac followed by BNT162b2 booster. c, Neutralization titers against the ancestral virus and the Delta and Omicron variants at 28 days after the booster in non-previously infected (left; ancestral, $n=75$; Delta, $n=75$; Omicron, $n=75$ ) and SARS-CoV-2-previously infected participants (right; ancestral, $n=26$; Delta, $n=26$; Omicron, $n=26$ ). Significance was measured using one-way ANOVA corrected using Tukey's test. Violin plots represent the mean \pm s.d. Dotted lines indicate the mean and are colored accordingly. d, Neutralization titers from the participants in $\mathbf{c}$ by SARS-CoV-2 infection status. Significance was measured using one-way ANOVA corrected using Tukey's test. (-) Vaccinated-uninfected, $n=57 ;(+)$ vaccinated-previously infected, $n=24$. e, Plasma neutralization titers at 28 days after the booster dose by time from the second CoronaVac vaccination ( $n=101$ in each group). $\mathbf{f}, \mathbf{g}$, Plasma neutralization titers measured before (f) and 28 days after the booster dose (g) by time from SARS-CoV-2 infection. Ancestral, $n=26 ;$ Delta, $n=26$; Omicron, $n=26$. The lines indicate the cross-sectional average from each group and the shading represents the $95 \% \mathrm{Cl}$. Each dot represents a single individual. A multivariable linear regression modeling was used to access significance. ${ }^{\star} P<0.05 ;{ }^{\star \star} P<0.01 ;{ }^{\star \star \star} P<0.001 ;{ }^{\star \star \star \star} P<0.0001$.

had received the CoronaVac-BNT162b2 regimen and those who had received the two-dose mRNA regimen, 28 days after the last shot (Fig. 1c,d).
Next, we extended our analysis to investigate potential neutralizing antibody escape against the Omicron variant after vaccination. In the HCW cohort we observed a 14.5-fold reduction in 
neutralization titers against the Omicron variant (Extended Data Fig. 1) at 28 days after the second vaccination dose (the peak of neutralization titers $\left.^{8}\right)$, measured by half-maximum plaque reduction neutralizing assays (PRNT50).

Booster doses of homologous mRNA vaccines have been shown to enhance neutralizing antibody response against the Omicron variant $^{9-14}$. To further assess neutralization activity in the heterologous vaccination regimen, we compared neutralization titers against the ancestral virus, the Delta and the Omicron variants before and after the BNT162b2 booster. Omicron was 7.1-fold less sensitive to neutralization than ancestral virus and 3.6-fold less sensitive than the Delta variant when assayed with plasma samples obtained 28 days after the BNT162b2 booster (Fig. 1e). Notably, plasma from those who received two doses of CoronaVac had no neutralizing antibodies to Omicron prior to the BNT162b2 booster (Fig. 1f). After the booster, $80 \%$ of CoronaVac-BNT162b2 recipients developed neutralizing antibody titers against the Omicron variant. The BNT162b2 booster resulted in a 1.4-fold increase in neutralization activity against Omicron compared with the two-dose mRNA vaccine, 28 days after the last shot (Fig. 1f). Despite this increase, neutralizing antibody titers were reduced by 7.1 -fold and 3.6-fold for Omicron compared with ancestral and Delta SARS-CoV-2, respectively.

Next, we categorized individuals by their SARS-CoV-2 infection status (that is, previously infected versus non-previously infected) and assessed their neutralization titers against Omicron after vaccination. In individuals who had received the two mRNA vaccine doses, the neutralizing antibody titers against variants of concern for previously infected individuals were higher than those for non-previously infected individuals, 28 days after the last shot, as previously described ${ }^{8}$. And neutralization titers against Omicron decreased by 17.3 -fold (compared with lineage A) in non-previously infected and by 10.7-fold in previously infected individuals who had received the two mRNA vaccine doses (Fig. 2a). Additionally, in individuals who received the two mRNA doses or the heterologous CoronaVac-BNT162b2 booster regimen, the neutralizing antibody titers were elevated for the Delta variant in previously infected individuals but not for Omicron (Fig. 2b-d).

Finally, we investigated whether the timing interval between vaccination doses and SARS-CoV-2 infection correlates with vaccine-induced IgG and PRNT50 levels. As previously described ${ }^{2}$, anti-RBD IgG titers and neutralizing antibodies waned over time after CoronaVac vaccination (Extended Data Fig. 2). Importantly, the time interval between the second vaccination dose and the booster shot did not affect neutralization titers against ancestral and SARS-CoV-2 variants, measured 28 days after the BNT162b2 booster (Fig. 2e). However, the time interval between previous SARS-CoV-2 infection and vaccination correlated with neutralization titers against the ancestral strain and the Delta variant: the longer the time interval, the higher the neutralization responses (Fig. 2f,g). Notably, in the temporal analysis the neutralization titers against Omicron do not show such a correlation, even after the booster shot (Fig. 2g). In multivariable linear regression modeling, previous COVID-19 infection increased $\log$ PRNT50 by 0.17 (95\%CI: $0.02-0.33 ; P=0.03$ ) for the ancestral strain, by 0.23 (95\%CI: $0.08-0.39 ; P=0.003$ ) for the Delta variant, and by 0.15 (95\%CI: -0.01 to 0.30 ; $P=0.06$ ) for the Omicron variant after controlling for time from the second dose of the vaccine to the booster dose and for sex. It should be noted that stratification by infection status limited the sample size, which could affect the precision of our estimates for temporal analysis. Together, these data suggest that previous SARS-CoV-2 exposure improves the production of neutralizing antibodies in vaccinated individuals for the ancestral strain and the Delta variant.

We found that a regimen of heterologous two-dose CoronaVac prime vaccination followed by a single BNT162b2 booster induces elevated virus-specific antibody levels and potent neutralization activity against the ancestral and Delta SARS-CoV-2 strains, resembling the titers obtained after two doses of mRNA vaccines. After the BNT162b2 booster shot, $80 \%$ of participants developed neutralizing antibodies against Omicron and the levels were 1.4-fold higher than in participants who received two doses of mRNA vaccines. However, the neutralization titers against Omicron were significantly reduced compared with the neutralizing antibody levels against the ancestral virus and the Delta variant after the booster, suggesting a greater risk of vaccine breakthrough infections. Clinical follow-up is needed to assess the risk of serious disease in these individuals.

In agreement with previous reports, our data show that the Omicron variant escapes neutralizing antibodies elicited by two mRNA vaccine doses or two CoronaVac vaccine doses ${ }^{9,12,14-16}$. Notably, none of the individuals who were fully vaccinated with CoronaVac had detectable neutralizing antibodies against the SARS-CoV-2 Omicron variant. Preliminary mRNA vaccine studies suggest that booster doses can enhance neutralizing antibody response against the Omicron variant and therefore booster doses should be recommended ${ }^{12,17}$. However, our data suggest that the Omicron variant may be associated with lower COVID-19 vaccine effectiveness against infection, even after a single heterologous booster (CoronaVac followed by BNT162b2) and even in previously infected individuals. Our findings have immediate implications for multiple countries that previously used a two-dose regimen of CoronaVac, and are of particular importance given the increasing global need for heterologous vaccine boosters as a relevant strategy to combat the impact of emerging variants in countries where inactivated vaccines have been the dominant product used.

\section{Online content}

Any methods, additional references, Nature Research reporting summaries, source data, extended data, supplementary information, acknowledgements, peer review information; details of author contributions and competing interests; and statements of data and code availability are available at https://doi.org/10.1038/ s41591-022-01705-6.

Received: 4 January 2022; Accepted: 18 January 2022; Published online: 20 January 2022

\section{References}

1. Jara, A. Effectiveness of an inactivated SARS-CoV-2 vaccine in Chile. N. Engl. J. Med., https://doi.org/10.1056/NEJMoa2107715 (2021).

2. Naaber, P. et al. Dynamics of antibody response to BNT162b2 vaccine after six months: a longitudinal prospective study. Lancet Reg. Health Eur. 10, 100208 (2021).

3. Pegu, A. et al. Durability of mRNA-1273 vaccine-induced antibodies against SARS-CoV-2 variants. Science 373, 1372-1377 (2021).

4. Goldberg, Y. et al. Waning immunity after the BNT162b2 vaccine in Israel. N. Engl. J. Med. 385, e85 (2021).

5. Pulliam, J. R. C. et al. Increased risk of SARS-CoV-2 reinfection associated with emergence of the Omicron variant in South Africa. Preprint at medRxiv https://doi.org/10.1101/2021.11.11.21266068 (2021).

6. Andrews, N. et al. Effectiveness of COVID-19 vaccines against the Omicron (B.1.1.529) variant of concern. Preprint at medRxiv https://doi.org/10.1101/ 2021.12.14.21267615 (2021)

7. Collie, S., Champion, J., Moultrie, H., Bekker, L.-G. \& Gray, G. Effectiveness of BNT162b2 vaccine against Omicron variant in South Africa. N. Engl. J. Med. https://doi.org/10.1056/NEJMc2119270 (2021).

8. Lucas, C. et al. Impact of circulating SARS-CoV-2 variants on mRNA vaccine-induced immunity. Nature 600, 523-529 (2021).

9. Pajon R. et al. SARS-CoV-2 Omicron variant neutralization after mRNA-1273 booster vaccination. N. Engl. J. Med. https://doi.org/10.1056/ NEJMc2119912 (2022)

10. Gruell, H. et al. mRNA booster immunization elicits potent neutralizing serum activity against the SARS-CoV-2 Omicron variant. Nat. Med. https:// doi.org/10.1038/s41591-021-01676-0 (2022). 
11. Basile, K. et al. Improved neutralization of the SARS-CoV-2 Omicron variant after Pfizer-BioNTech BNT162b2 COVID-19 vaccine boosting. Preprint at bioRxiv https://doi.org/10.1101/2021.12.12.472252 (2021).

12. Garcia-Beltran, W. F. et al. mRNA-based COVID-19 vaccine boosters induce neutralizing immunity against SARS-CoV-2 Omicron variant. Cell https://doi.org/10.1016/j.cell.2021.12.033 (2022).

13. Schmidt, F. et al. Plasma neutralization of the SARS-CoV-2 Omicron variant. N. Engl. J. Med. https://doi.org/10.1056/NEJMc2119641 (2022).

14. Cele, S. et al. Omicron extensively but incompletely escapes Pfizer BNT162b2 neutralization. Nature https://doi.org/10.1038/s41586-02104387-1 (2021)

15. Nemet, I. et al. Third BNT162b2 vaccination neutralization of SARS-CoV-2 Omicron infection. N. Engl. J. Med. https://doi.org/10.1056/NEJMc2119358 (2021).

16. Lu, L. et al. Neutralization of SARS-CoV-2 Omicron variant by sera from BNT162b2 or Coronavac vaccine recipients. Clin. Infect. Dis. ciab1041 https://doi.org/10.1093/cid/ciab1041 (2021).
17. Kalinich, C. C. et al. Real-time public health communication of local SARS-CoV-2 genomic epidemiology. PLoS Biol. 18, e3000869 (2020).

Publisher's note Springer Nature remains neutral with regard to jurisdictional claims in published maps and institutional affiliations.

(c) (i) Open Access This article is licensed under a Creative Commons

Attribution 4.0 International License, which permits use, sharing, adaptation, distribution and reproduction in any medium or format, as long as you give appropriate credit to the original author(s) and the source, provide a link to the Creative Commons license, and indicate if changes were made. The images or other third party material in this article are included in the article's Creative Commons license, unless indicated otherwise in a credit line to the material. If material is not included in the article's Creative Commons license and your intended use is not permitted by statutory regulation or exceeds the permitted use, you will need to obtain permission directly from the copyright holder. To view a copy of this license, visit http://creativecommons. org/licenses/by/4.0/.

(C) The Author(s) 2022 


\section{Methods}

Ethics statement. This study was approved by the National Bioethics Committee of the Dominican Republic (CONABIOS). The participants received two doses of the inactivated whole-virion vaccine CoronaVac followed by a BN162b2 booster dose at least 4 weeks after the second dose of CoronaVac. The Dominican Republic initiated the COVID-19 vaccination and booster campaigns in February and July 2021, respectively. All Dominican Republic participants provided consent to enroll in this observational study. The mRNA vaccine BNT162b2 booster was given between 30 July and 27 August 2021. HCW volunteers from the Yale-New Haven Hospital (YNHH) were enrolled and included in this study (Institutional Review Board protocol ID 2000028924, approved by the Yale Human Research Protection Program Institutional Review Board and the Yale School of Medicine). The HCW volunteers received the mRNA vaccine (100 $\mu \mathrm{g}$ mRNA-1273, Moderna or $20 \mu \mathrm{g}$ BNT162b2, Pfizer-BioNTech) between November 2020 and January 2021. Informed consent was obtained from all enrolled. None of the participants had serious adverse effects after vaccination.

Vaccinated volunteers. A total of 101 volunteers from the Dominican Republic and $37 \mathrm{HCW}$ participants from the $\mathrm{YNHH}$ were followed serially after vaccination. For the Dominican Republic cohort, plasma samples were collected at baseline (before the booster, after two doses), and at 7 and 28 days after the booster (third dose). Plasma from the HCWs included in this study was collected 28 days after the second vaccination dose. Demographic information was aggregated through a systematic review and was used to construct Extended Data Tables 1 and 2. The clinical data were collected using REDCap (v5.19.15, (C2021 Vanderbilt University). Blood collection was performed and recorded by a separate team. Participants clinical information and time points of collection was not available until after the raw data had been processed and analyzed. ELISA and neutralizations were performed blinded. Prior SARS-CoV-2 infection was confirmed using ELISA and/or a polymerase chain reaction test. Documented history of vaccination and vaccination date were evidenced by the official vaccination card. The time window of previous COVID-19 infection is based on participant self-report. The time window after CoronaVac vaccination and the infection status, indicated in days post vaccination or COVID-19 exposure are available in Source Data 1.

Plasma isolation and storage. Whole blood was collected in heparinized CPT blood vacutainers (BD Biosciences, cat. no. BDAM362780) and kept on gentle agitation until processing. All blood was processed on the day of collection in a single-step standardized method. Plasma samples were collected after centrifugation of whole blood at $600 \times g$ for $20 \mathrm{~min}$ at room temperature without brake. The undiluted plasma was transferred to $15 \mathrm{ml}$ polypropylene conical tubes, and aliquoted and stored at $-80^{\circ} \mathrm{C}$ for subsequent shipping and analysis. Plasma samples were collected from Dominican Republican participants and shipped to Yale University. The plasma was aliquoted and heat-inactivated at $56^{\circ} \mathrm{C}$ for $30 \mathrm{~min}$ to inactivate complement prior to micro-neutralization.

SARS-CoV-2 culture. TMPRSS2-Vero E6 kidney epithelial cells were cultured in DMEM supplemented with $1 \%$ sodium pyruvate (NEAA) and $10 \%$ fetal bovine serum (FBS) at $37^{\circ} \mathrm{C}$ and $5 \% \mathrm{CO}_{2}$. The cell line had tested negative for contamination with mycoplasma. SARS-CoV-2 lineage A (USA-WA1/2020) was obtained from BEI Resources (cat. no. NR-52281). Alpha, Beta, Gamma, Delta and Omicron variants were isolated from nasopharyngeal specimens. Alpha, Beta, Gamma and Delta SARS-CoV-2 samples were sequenced as part of the Yale Genomic Surveillance Initiative's weekly surveillance program in Connecticut, United States $^{17}$. Omicron (lineage BA.1) was sequenced by the Connecticut State Department of Public Health (GISAID accession: EPI_ISL_7313633). The isolates were cultured and resequenced as previously described ${ }^{8,18}$. In brief, samples were filtered through a $45 \mu \mathrm{M}$ filter and serially diluted from 1:50 to 1:19,531,250. The dilution was subsequently incubated with TMPRSS2-Vero E6 cells in a 96-well plate and adsorbed for $1 \mathrm{~h}$ at $37^{\circ} \mathrm{C}$. After adsorption, replacement medium was added, and cells were incubated at $37^{\circ} \mathrm{C}$ for up to 5 days. Supernatants from cell cultures with cytopathic effect were collected, frozen, thawed and analyzed using RT-qPCR. Fresh cultures were inoculated with the lysates as described above for viral expansion. Nucleic acid was extracted using the ThermoFisher MagMAX viral/pathogen nucleic acid isolation kit and libraries were prepared using the Illumina COVIDSeq Test (RUO version). Pooled libraries were sequenced on the Illumina NovaSeq (paired-end 150) by the Yale Center for Genome Analysis. Data were analyzed and consensus genomes were generated using iVar (v1.3.1). The resequenced genomes were submitted to the National Center for Biotechnology Information (NCBI; GenBank accession numbers: ancestral lineage A, MZ468053; Alpha, MZ202178; Beta, MZ468007; Gamma, MZ202306; Delta, MZ468047; Omicron, OL965559). The pelleted virus was then resuspended in PBS and aliquoted for storage at $-80^{\circ} \mathrm{C}$. Viral titers were measured by standard plaque assay using TMPRSS2-Vero E6 cells. In brief, $300 \mu \mathrm{l}$ serial fold virus dilutions were used to infect Vero E6 cells in MEM supplemented with $\mathrm{NaHCO}_{3}, 4 \%$ FBS and $0.6 \%$ Avicel RC-581. Plaques were resolved at $48 \mathrm{~h}$ after infection by fixing in $10 \%$ formaldehyde for $1 \mathrm{~h}$ followed by staining with $0.5 \%$ crystal violet in $20 \%$ ethanol. Plates were rinsed in water to plaques quantification. All experiments were performed in a biosafety level 3 laboratory with approval from the Yale Environmental Health and Safety office.
SARS-CoV-2-specific antibody measurements. ELISAs were performed as previously described ${ }^{8}$. In brief, Triton X-100 and RNase A were added to serum samples at final concentrations of $0.5 \%$ and $0.5 \mathrm{mg} \mathrm{ml}^{-1}$, respectively, and incubated at room temperature for $30 \mathrm{~min}$ before use, to reduce the risk from any potential virus in serum. The 96-well MaxiSorp plates (Thermo Scientific, cat. no. 442404) were coated with $50 \mu \mathrm{l}$ per well recombinant SARS CoV-2 total spike protein (ACROBiosystems, cat. no. SPN-C52H9-100 $\mu$ g) or RBD (ACROBiosystems, cat. no. SPD-C52H3-100 $\mu \mathrm{g}$ ) at a concentration of $2 \mu \mathrm{g} \mathrm{ml}^{-1}$ in PBS and incubated overnight at $4{ }^{\circ} \mathrm{C}$. The coating buffer was removed, and plates were incubated for $1 \mathrm{~h}$ at room temperature with $200 \mu \mathrm{l}$ blocking solution (PBS with $0.1 \%$ Tween-20, $3 \%$ milk powder). Plasma was diluted serially $1: 100,1: 200,1: 400$ and 1:800 in dilution solution (PBS with $0.1 \%$ Tween-20, $1 \%$ milk powder), and $100 \mu$ l diluted serum was added for $2 \mathrm{~h}$ at room temperature. Human Anti-Spike (SARS-CoV-2 Human Anti-Spike, AM006415) (Active Motif no. 91351) was serially diluted to generate a standard curve, starting at a concentration of $1,000 \mathrm{ng} \mathrm{ml}^{-1}$. Plates were washed three times with PBS-T (PBS with $0.1 \%$ Tween-20) and then $50 \mu \mathrm{l}$ HRP anti-Human IgG Antibody (GenScript A00166, 1:5,000) diluted in dilution solution was added to each well. After $1 \mathrm{~h}$ of incubation at room temperature, plates were washed six times with PBS-T. Plates were developed with $100 \mu \mathrm{l}$ TMB Substrate Reagent Set (BD Biosciences, cat. no. 555214) and the reaction was stopped after $5 \mathrm{~min}$ by the addition of $2 \mathrm{~N}$ sulfuric acid. Plates were then read at a wavelength of $450 \mathrm{~nm}$ and $570 \mathrm{~nm}$.

Neutralization assay. Sera from vaccinated individuals were heat treated for $30 \mathrm{~min}$ at $56^{\circ} \mathrm{C}$. Sixfold serially diluted plasma, from 1:10 to 1:2,430, was incubated with SARS-CoV-2 variants for $1 \mathrm{~h}$ at $37^{\circ} \mathrm{C}$. The mixture was subsequently incubated with TMPRSS2-Vero E6 cells in a 12-well plate for $1 \mathrm{~h}$ for adsorption. The cells were then overlayed with MEM supplemented with $\mathrm{NaHCO}_{3}, 4 \%$ FBS and $0.6 \%$ Avicel. Plaques were resolved at $40 \mathrm{~h}$ after infection by fixing in $10 \%$ formaldehyde for $1 \mathrm{~h}$ followed by staining in $0.5 \%$ crystal violet. All experiments were performed in parallel with baseline control sera, in an established viral concentration to generate 60-120 plaques per well.

Statistical analysis. All analyses of patient samples were conducted using GraphPad Prism 8.4.3 and JMP 15. Multiple group comparisons were analyzed by running parametric (ANOVA) statistical tests. Multiple comparisons were corrected using Tukey's and Dunnett's tests as indicated in the figure legends. We used a multivariable linear regression model with $\log$ PRNT50 as the dependent variable, and previous COVID-19 infection and time from the second dose of the vaccine to the booster dose (in days) as the explanatory variables to determine the effect of previous COVID-19 infection.

Reporting Summary. Further information on research design is available in the Nature Research Reporting Summary linked to this article.

\section{Data availability}

All of the background participant information and data generated in this study are included in Source Data 1. The genome information and aligned consensus genomes for SARS-CoV-2 variants used in this study are available at NCBI (GenBank accession numbers: ancestral lineage A, MZ468053; Alpha, MZ202178; Beta, MZ468007; Gamma, MZ202306; Delta, MZ468047; Omicron, OL965559). Additional correspondence and requests for material should be addressed to the corresponding authors. Source data are provided with this paper.

\section{References}

18. Mao, T. et al. A stem-loop RNA RIG-I agonist protects against acute and chronic SARS-CoV-2 infection in mice. J. Exp. Med. 219, e20211818 (2022).

\section{Acknowledgements}

We thank the study participants who donated specimens for this study. We also thank M. Linehan for technical and logistical assistance and D. Mucida for discussions. This work was funded by the Government of the Dominican Republic and supported by the Dominican National Health Cabinet as well as the Ministry of Health. The Dominican Republic team thanks M. Caram (Profamilia) and M. Kelly (Laboratorio de Referencia) for their contributions to the set up of the study platform. The study was also supported by the Women's Health Research at Yale Pilot Project Program (A.I.), Fast Grant from Emergent Ventures at the Mercatus Center (A.I. and N.D.G.), Mathers Foundation, and the Ludwig Family Foundation, the Department of Internal Medicine at the Yale School of Medicine, Yale School of Public Health and the Beatrice Kleinberg Neuwirth Fund. A.I. is an Investigator of the Howard Hughes Medical Institute. C.L. is a Pew Latin American Fellow. V.S.M. is supported by the CAPES-YALE fellowship.

\section{Author contributions}

E.P.-T., C.L., V.S.M., M.M., V.B., S.H.V., N.D.G. and A.I. conceived the study. S.B.O., A.I.K. and I.Y. designed and implemented the HCW cohort study. V.B., L.C., E.D.I.C., A.J., M.D.I.S., I.Y. and P.L. collected and processed the plasma samples. C.L. and V.S.M. performed SARS-CoV-2-specific antibody ELISAs and the neutralization assays. V.B., L.C., M.C. and I.Y. collected epidemiological and clinical data. C.B.F.V., M.I.B., 
K.B., C.P., R.D., E.G., A.M. and J.R. surveilled, detected and performed virus sequencing. C.L. and V.S.M. isolated the SARS-CoV-2 variants. V.B., I.Y. and M.C. assisted in the volunteers' identification and enrollment. C.L. and V.S.M. analyzed the data. A.A.M. assisted in the longitudinal analysis. E.P.-T. and M.M. designed, managed and monitored the quality of the epidemiological data collected in the REDCap database. C.L., E.P.-T. and A.I. drafted the manuscript. All authors reviewed and approved the manuscript. E.P.-T. and A.I. secured funds and supervised the project.

\section{Competing interests}

A.I. served as a consultant for RIGImmune, Xanadu and Revelar Biotherapeutics.

I.Y. reported being a member of the mRNA-1273 Study Group and has received funding to her institution to conduct clinical research from BioFire, MedImmune, Regeneron, PaxVax, Pfizer, GSK, Merck, Novavax, Sanofi-Pasteur and Micron. N.D.G. is a consultant for Tempus Labs to develop infectious disease diagnostic assays. A.I.K. serves as an expert panel member for Reckitt Global Hygiene Institute, a scientific advisory board member for Revelar Biotherapeutics and a consultant for Tata Medical and Diagnostics and Regeneron Pharmaceuticals, and has received grants from Merck, Regeneron
Pharmaceuticals, and Tata Medical and Diagnostics for research related to COVID-19 outside of the submitted work. All other authors declare no competing interests.

\section{Additional information}

Extended data are available for this paper at https://doi.org/10.1038/s41591-02201705-6.

Supplementary information The online version contains supplementary material available at https://doi.org/10.1038/s41591-022-01705-6.

Correspondence and requests for materials should be addressed to Carolina Lucas or Akiko Iwasaki.

Peer review information Nature Medicine thanks Deepta Bhattacharya and the other anonymous, reviewer(s) for their contribution to the peer review of this work. Saheli Sadanand was the primary editor on this article and managed its editorial process and peer review in collaboration with the rest of the editorial team.

Reprints and permissions information is available at www.nature.com/reprints. 


\section{Plaque reduction half-maximal neutralizing titer (PRNT50) against VOCs}

A) $\quad$ mRNAVac $(2 x)$ Yale (28 Days)

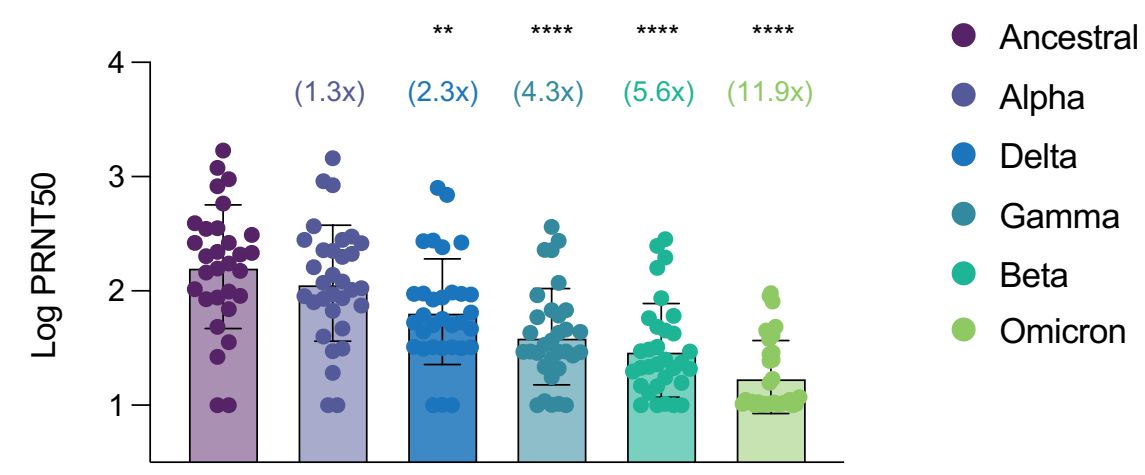

Extended Data Fig. 1 | Impact of SARS-CoV-2 Omicron on neutralization capacity of in mRNA-vaccinated participants. Plasma neutralization titers against ancestral lineage A virus, (WA1, USA) and VOCs: Alpha, Beta, Delta and Omicron. SARS-CoV-2 variants were isolated from nasopharyngeal swabs of infected individuals and ancestral (WA1, USA) isolate was obtained from BEI. Neutralization capacity was accessed using plasma samples from HCW participants that received 2 doses of the mRNA vaccine (mRNAVac (2x) Yale), 28 days post second vaccination dose at the experimental sixfold serial dilutions (from 1:3 to 1:2430). Significance was assessed by One-way ANOVA corrected for multiple comparisons using Dunnett's method. Neutralization capacity to the variants was compared to neutralization capacity against the ancestral strain. Boxes represent mean values \pm standard deviations. The numbers in parentheses indicate the median fold change in neutralization resistance for the indicated variants compared to ancestral strain. $\mathrm{n}=32 /$ group Each dot represents a single individual. ${ }^{\star \star \star \star} p<0.0001^{\star \star} p<0.005$. 


\section{Previous to booster (Baseline) - CoronaVac (2x) DR}

A)

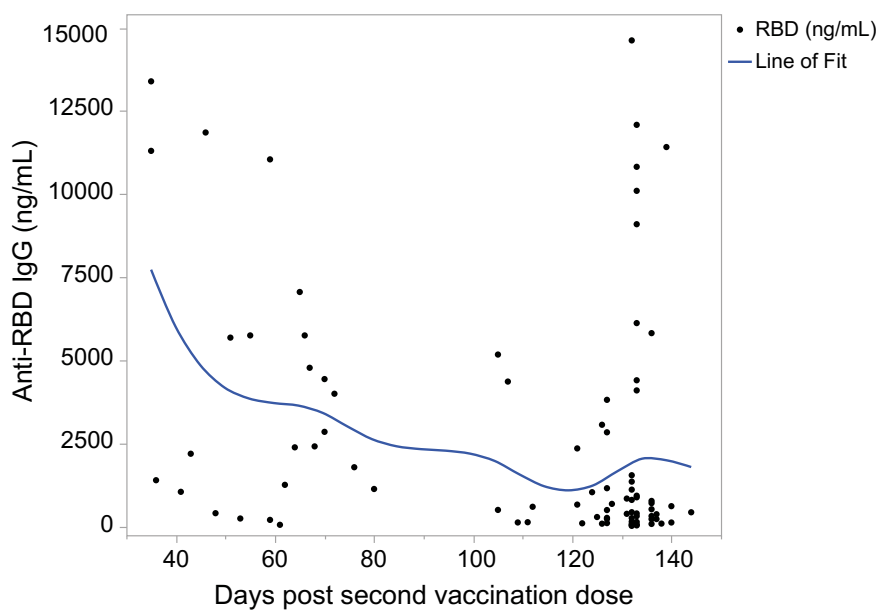

B)

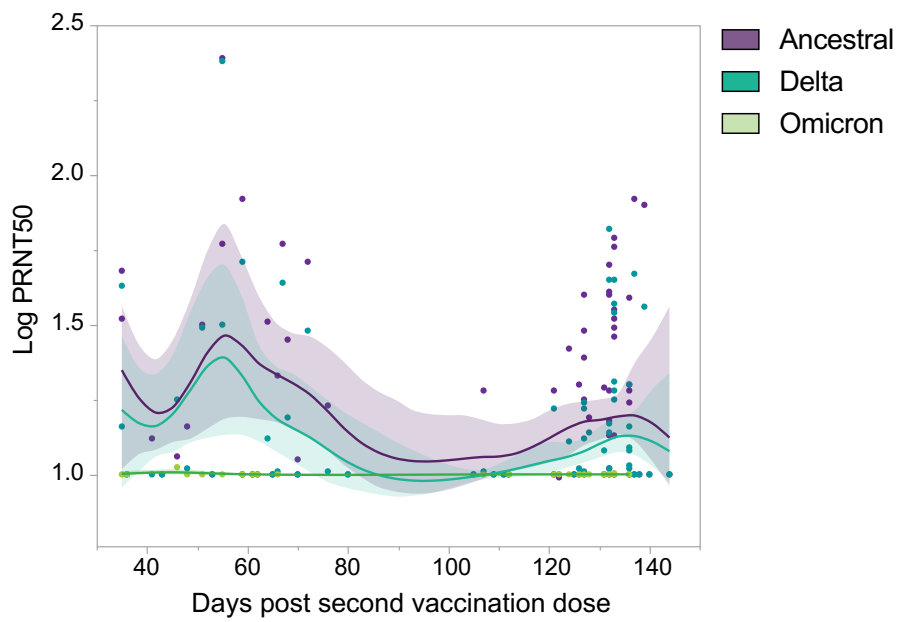

Extended Data Fig. 2 | Plasma antibody kinetics post CoronaVac vaccination. a, Vaccinated participants plasma reactivity to RBD measured by ELISA. b, Plasma neutralization titers measured at baseline, previously to BNT162b2 booster shot against ancestral virus, Delta and Omicron variants. Regression lines are shown over time as days post 2 CoronaVac dose vaccination, as purple (ancestral), dark green (Delta) and light green (Omicron). Lines indicates cross-sectional averages from each group, with shading representing $95 \% \mathrm{Cl}$ and colored accordingly. Each dot represents a single individual. $n=101$ (Ancestral); $n=101$ (Delta); $n=80$ (Omicron). 
Extended Data Table 1 | Patient data: Dominican Republic SARS-CoV-2-vaccinated cohort

Dominican Republic Cohort

\begin{tabular}{lcccc} 
Individual variables & N & (\%) & Mean & SD \\
\hline Age & 101 & & 40.4 & 13.4 \\
Gender & & & & \\
$\quad$ Male & 31 & 30.7 & & \\
$\quad$ Female & 70 & 69.3 & & \\
BMI & 101 & & 27.63 & 5.53 \\
Infection Status & & & & \\
$\quad$ Non-previous infected & 75 & 74.25 & & \\
$\quad$ Previous infected & 26 & 25.74 & & \\
$\quad$ Days since last infection & & & 326.26 & 131.01 \\
Days since last CoronaVac dose & 101 & & 113 & 33
\end{tabular}

Exact counts for each demographic category are displayed in $\mathrm{N}$ cell. Percentages of total, where applicable, are displayed in (\%) cell. The mean with accompanying standard deviations for each measurement are displayed in Mean and SD cells respectively. 
Extended Data Table 2 | Patient data: Yale healthcare worker SARS-CoV-2-vaccinated cohort HCWs-Yale Cohort

\begin{tabular}{lcccc} 
Individual variables & N & (\%) & Mean & SD \\
\hline Age & 32 & & 44.03 & 12.7 \\
$\quad$ Gender & & & & \\
$\quad$ Male & 5 & 15.6 & & \\
$\quad$ Female & 27 & 84.3 & & \\
Infection Status & & & \\
$\quad$ Non-previous infected & 17 & 53.1 & \\
$\quad$ Previous infected & 15 & 46.8 & \\
$\quad$ Vaccine & & & \\
$\quad$ BNT162b2 & 7 & 21.8 & \\
$\quad$ mRNA-1273 & 25 & 78.1 &
\end{tabular}

Exact counts for each demographic category are displayed in $\mathrm{N}$ cell. Percentages of total, where applicable, are displayed in (\%) cell. The mean with accompanying standard deviations for each measurement are displayed in Mean and SD cells respectively. 


\section{nature portfolio}

Corresponding author(s): Akiko Iwasaki

Last updated by author(s): Jan 13, 2022

\section{Reporting Summary}

Nature Portfolio wishes to improve the reproducibility of the work that we publish. This form provides structure for consistency and transparency in reporting. For further information on Nature Portfolio policies, see our Editorial Policies and the Editorial Policy Checklist.

\section{Statistics}

For all statistical analyses, confirm that the following items are present in the figure legend, table legend, main text, or Methods section.

$\mathrm{n} / \mathrm{a}$ Confirmed

$\bigotimes$ The exact sample size $(n)$ for each experimental group/condition, given as a discrete number and unit of measurement

Х $\square$ A statement on whether measurements were taken from distinct samples or whether the same sample was measured repeatedly

The statistical test(s) used AND whether they are one- or two-sided

Only common tests should be described solely by name; describe more complex techniques in the Methods section.

$\bigotimes$ A description of all covariates tested

$\bigotimes$ A description of any assumptions or corrections, such as tests of normality and adjustment for multiple comparisons

A full description of the statistical parameters including central tendency (e.g. means) or other basic estimates (e.g. regression coefficient) AND variation (e.g. standard deviation) or associated estimates of uncertainty (e.g. confidence intervals)

For null hypothesis testing, the test statistic (e.g. $F, t, r$ ) with confidence intervals, effect sizes, degrees of freedom and $P$ value noted Give $P$ values as exact values whenever suitable.

Х $\square$ For Bayesian analysis, information on the choice of priors and Markov chain Monte Carlo settings

$\bigotimes \square$ For hierarchical and complex designs, identification of the appropriate level for tests and full reporting of outcomes

$\triangle \square$ Estimates of effect sizes (e.g. Cohen's $d$, Pearson's $r$ ), indicating how they were calculated

Our web collection on statistics for biologists contains articles on many of the points above.

\section{Software and code}

Policy information about availability of computer code

Data collection REDCap (v5.19.15 @2021 Vanderbilt University) (clinical data aggregation).

Data analysis Imp Pro 15.0.0 (SAS Institute) (graphs/statistics), and GraphPad Prism 8.4.3(graphs/statistics).

For manuscripts utilizing custom algorithms or software that are central to the research but not yet described in published literature, software must be made available to editors and

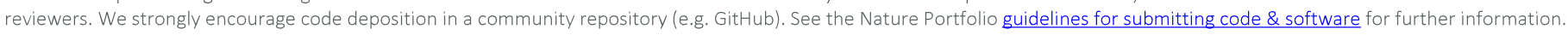

\section{Data}

Policy information about availability of data

All manuscripts must include a data availability statement. This statement should provide the following information, where applicable:

- Accession codes, unique identifiers, or web links for publicly available datasets

- A description of any restrictions on data availability

- For clinical datasets or third party data, please ensure that the statement adheres to our policy

All the background information of participants and data generated in this study are included in Source Data1. The genome information and aligned consensus genomes for SARS-CoV-2 variants used in this study are available on NCBI (GenBank Accession numbers: ancestral lineage A= MZ468053, Alpha = MZ202178, Beta = MZ468007, Gamma = MZ202306, Delta = MZ468047, Omicron = OL965559). Additional correspondence and requests for materials should be addressed to the corresponding authors. 
Please select the one below that is the best fit for your research. If you are not sure, read the appropriate sections before making your selection.

\ Life sciences

Behavioural \& social sciences

Ecological, evolutionary \& environmental sciences

For a reference copy of the document with all sections, see nature.com/documents/nr-reporting-summary-flat.pdf

\section{Life sciences study design}

All studies must disclose on these points even when the disclosure is negative.

Sample size No statistical methods were used to calculate the sample size. Sample size was determined based on the number of adults ( $\geq 18$ years old) from the Dominican Republic that received the mRNA vaccine, Pfizer as a booster and consent in participating in the current study. Republic initiated the COVID-19 vaccination and booster campaigns in February and July 2021, respectively. mRNA vaccine BNT162b2 booster were administrated between July 30 and August 27, 2021. This study enrolled 103 volunteers and was approved by the National Bioethics Committee of the Dominican Republic (CONABIOS). Informed consent was obtained by trained staff and sample collection commenced immediately upon study enrollment. Participants were followed serially post-vaccination. Clinical specimens were collected at baseline (previous to booster), 7- and 28- post third vaccination dose. Alpha, Beta, Gamma, Delta and Omicron variants were isolated from nasopharyngeal specimens from the Yale SARS-CoV-2 Genomic Surveillance Initiative's weekly surveillance program in Connecticut, US.

Data exclusions Twp participant that received an adenovirus- based vaccine were excluded from this study. For the current study we had only included participants that received CoronaVac and Pfizer vaccines as booster shot.

Replication ELISAs were done in duplicate for each sample. Neutralization assays were done with 6 fold dilution for each sample to generate a curve and access the IC50 values. For all assays, longitudinal analyses were performed from human individuals samples. All replications were successful.

Randomization Vaccinated donors were stratified in two major groups, previously infected with SARS-CoV2 (recovered) on uninfected (naive), confirmed by serology.

Blinding The clinical data were collected using REDCap software. Blood acquisition was performed and recorded by a separate team. Clinical information and time points of collection information was not available until after processing and analyzing raw data. ELISA and neutralizations were blinded.

\section{Reporting for specific materials, systems and methods}

We require information from authors about some types of materials, experimental systems and methods used in many studies. Here, indicate whether each material, system or method listed is relevant to your study. If you are not sure if a list item applies to your research, read the appropriate section before selecting a response.

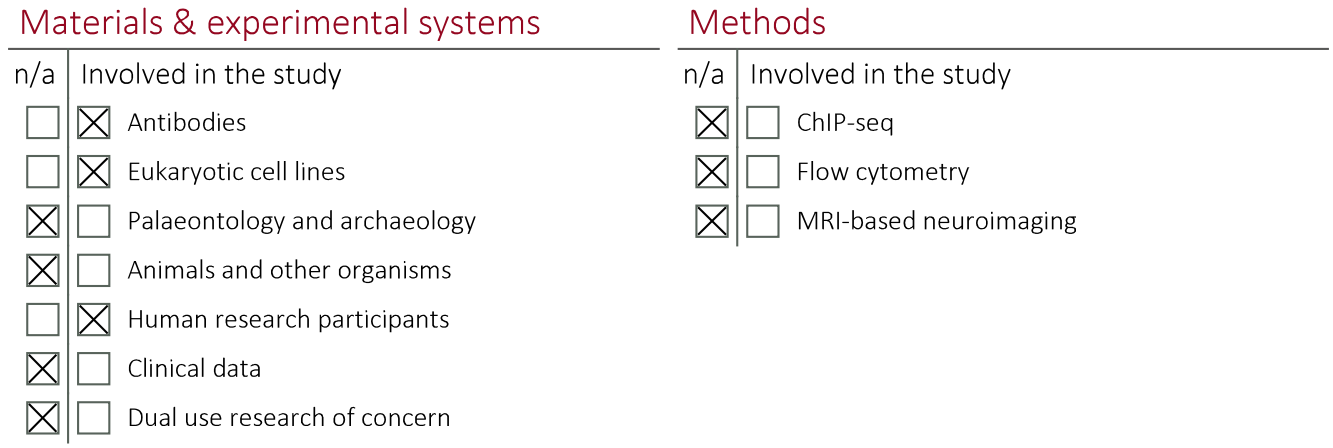

\section{Antibodies}

Antibodies used

Validation
All antibodies used in this study are against human proteins:

Human Anti-Spike (SARS-CoV-2 Human Anti-Spike (AM006415) (Active Motif \#91351) was serially diluted to generate a standard curve. HRP anti-Human IgG Antibody (GenScript \#A00166, 1:5,000.

The antibodies used in this study are commercially available, and all have been validated by the manufacturers and used by other publications. Likewise, we titrated these antibodies according to our own our staining conditions.

Human Anti-Spike (SARS-CoV-2 Human Anti-Spike (Clone: AM006415, 415-6)) (Active Motif \#91351) : this antibody has been tested by ELISA and is specific for SARS-CoV-2 Spike protein S1 Subunit of Receptor Binding Domain (RBD). Details on the development and characterization of this clone are available here: Wan J., et. al. Human IgG neutralizing monoclonal antibodies block SARS-CoV-2 infection. Cell Reports, 32: 107918, July 21, 2020.

HRP anti-Human IgG Antibody: this antibody is purified by immunoaffinity chromatography and then conjugated to horseradish peroxidase. Species Reactivity: human 
Policy information about cell lines

Cell line source(s)

TMPRSS2-VeroE6 kidney epithelial cell line was obtained from the ATCC

\section{Authentication}

Mycoplasma contamination

Commonly misidentified lines (See ICLAC register)
TMPRSS2-VeroE6 was obtained from ATCC, tested and authenticated by morphology, karyotyping, and PCR based approaches.

The cell line tested negative for contamination with mycoplasma.

No commonly misidentified cell lines were used in the study

\section{Human research participants}

Policy information about studies involving human research participants

Population characteristics

Recruitment

Ethics oversight
Cohort characteristics are available in Table 1.

Participants were recruited between February and July 2021. mRNA vaccine BNT162b2 booster were administrated between July 30 and August 27, 2021 as part of the Republic Dominican COVID-19 vaccination booster campaigns Participants were enrollment with no self selection. Informed consent was obtained by trained staff and sample collection commenced immediately upon study enrollment. Plasma samples were collected at baseline (prior to booster, after two doses), 7 and 28 days after the booster (third dose) administration.

Health care worker (HCW) volunteers from the Yale New Haven Hospital (YNHH) were enrolled and included in this study. The HCWs volunteers received the mRNA vaccine (100 micrograms mRNA-1273, Moderna or 20 micrograms, BNT162b2, Pfizer-BioNTech) between November 2020 and January 2021. Plasma samples were collected at baseline (prior to vaccination, 7 and 28 days after the first and second vaccination dose. Participants were enrollment with no self selection. Informed consent was obtained by trained staff.

Yale Human Research Protection Program Institutional Review Boards. Informed consents were obtained from all enrolled healthcare workers. - Our research protocol was reviewed and approved by the Yale School of Medicine IRB Protocol ID 2000028924. This study was approved by the National Bioethics Committee of the Dominican Republic (CONABIOS). Informed consent was obtained by trained staff and records maintained in our research database for the duration of our study. There were no minors included on this study. 